\title{
EFEITOS DE COMPLEXIDADE DE TAREFAS SOBRE O COMPORTAMENTO PRECORRENTE AUXILIAR NO TREINO E RECOMBINAÇÃO
}

\section{EFFECTS OF TASK COMPLEXITY ON AUXILIARY PRECURRENT BEHAVIOR DURING TRAINING AND GENERALIZATION SESSIONS}

\author{
Domingos Sávio Coelho ${ }^{1}$ e Jorge M. Oliveira-Castro \\ UNIVERSIDADE DE BRASÍLIA, BRASIL
}

\begin{abstract}
RESUMO
Comportamentos precorrentes auxiliares (e.g., consultar uma agenda para discar um número de telefone) tornam mais provável o reforço da resposta final de uma sequência, apesar de não serem exigidos pelas contingências. Com o objetivo de investigar os possíveis efeitos de mudanças na complexidade da tarefa sobre a diminuição de tais comportamentos, um experimento utilizando uma tarefa de pares associados formados por caracteres arbitrários foi conduzido. Doze estudantes universitários deveriam aprender o segundo membro do par ao ser apresentado o primeiro membro, sendo que para isso podiam consultar uma tela de auxílio (precorrente auxiliar) contendo o segundo membro. O objetivo da tarefa era ser capaz de digitar o segundo membro sem consulta ao auxílio. Em uma das condições (Prob $1,00)$ as características do primeiro membro do par associado prediziam as características do segundo membro, enquanto em outra condição (Prob 0,33) isso não ocorria. A duração da resposta precorrente auxiliar foi sistematicamente menor na condição Prob 1,00 do que na Prob 0,33 e nas sessões de recombinação do que nas de treino. Tais resultados sugerem um modelo de análise para explicar, por exemplo, alguns efeitos de irregularidades entre grafemas e fonemas no ensino de leitura.
\end{abstract}

Palavras-chave: complexidade da tarefa, generalização recombinativa, comportamento precorrente auxiliar

\begin{abstract}
Auxiliary precurrent behaviors (e.g., looking up a directory in order to dial a phone number) increase the likelihood of reinforcement for the final response of a sequence. These precurrents are not required by the contingency. With the purpose of investigating the possible effects of changes in task complexity upon the decrease of such behaviors, an experiment using a paired-associates task, formed by arbitrary characters, was conducted. Twelve undergraduate students should learn the second member of the associated pair, upon being presented with the first, in a situation in which they could look up an auxiliary screen (auxiliary precurrent response) containing the second member. The purpose of the task was to be able to type the second member without looking up the auxiliary screen. In one Condition (Prob 1,00) the characteristics of the first member of the associated pair predicted the characteristics of the second, whereas in the other condition (Prob 0,33) this did not occur. The auxiliary precurrent duration was systematically shorter in Condition Prob 1,00 than in Condition Prob 0,33 and in recombination than in training sessions. Such results suggest, for example, some effects of letter and phoneme irregularities in teaching to read.
\end{abstract}

Key words: task complexity, recombinative generalization, precurrent auxiliary behavior 
Comportamento precorrente auxiliar é definido como passos necessários que tornam mais provável o reforço da resposta final. Por exemplo, realizar uma tarefa escolar de multiplicação pode envolver a emissão de precorrentes como escrever no papel, consultar a tabuada etc. Tais precorrentes são denominados auxiliares porque não são exigidos pela contingência, isto é, a resposta final (o resultado da multiplicação) pode ocorrer e ser reforçada sem a emissão de tais precorrentes (Oliveira-Castro, Coelho, \& Oliveira-Castro, 1999). Segundo Oliveira-Castro et al. (1999), o decréscimo do comportamento precorrente auxiliar em função das tentativas pode ser descrito pela Equação:

$$
\text { Duração/correta }=b-a \log (\text { tentativas })
$$

onde a duração da resposta precorrente por corretas é uma função linear do logaritmo do número de tentativas; sendo $b$ e $a$ parâmetros derivados empiricamente. A área da função derivada da Equação $1, b^{2} / 2 a$, pode ser concebida como uma medida global de desempenho ao dar a soma estimada de duração da resposta precorrente por resposta correta.

A complexidade da tarefa é uma das variáveis que pode afetar a diminuição de tais comportamentos precorrentes. Contudo, as tentativas de classificação de tarefas em psicologia impossibilitam a generalização dos resultados para novas tarefas por serem excessivamente detalhadas ou amplas; classificaçōes intermediárias (e.g., a taxonomia de Gagné) carecem de validade (Fleishman, 1975; Wood, 1986).

Segundo Oliveira-Castro et al. (1999), o nível de desempenho do indivíduo em qualquer tarefa é o produto da interação entre certas características da tarefa e o repertório anterior deste indivíduo; o uso do predicado "difícil" ilustra tal interação: o predicado "difícil" se refere a níveis inferiores de desempenho; neste caso, tarefa difícil seria aquela que poucos realizam bem. Segundo OliveiraCastro et al. (1999), a complexidade da tarefa estaria relacionada a características da tarefa e não a habilidades do indivíduo, pois a complexidade da tarefa independe de quem a executa e não se altera após o indivíduo adquirir a habilidade de executá-la.

Segundo Oliveira-Castro et al. (1999), considerando que qualquer tarefa especifica quais respostas serão corretas (ou reforçadas) em quais situações (estímulos discriminativos), um modo possível de analisar complexidade de tarefas é interpretar qualquer tarefa como um conjunto de contingências programadas de reforço. A quantificação de tais contingências proporcionaria uma medida de complexidade de tarefas. Na tarefa de par associado, as contingências especificam que uma dada resposta (digitar um caractere) é correta somente se ocorrer na presença da forma correta e na posição correta. Forma e posição são duas dimensões funcionais na tarefa porque a consequência para cada resposta (certo ou errado) pode mudar dependendo destas dimensôes. A complexidade da tarefa pode ser manipulada por par (número de diferentes formas arbitrárias que formam cada conjunto de cinco dígitos), por posição (número de diferentes formas arbitrárias ocorrendo em cada uma das cinco posiçôes do segundo membro dos pares), e no conjunto total do material (número de diferentes formas arbitrárias que formam todos os pares e posições). Por exemplo, a complexidade dos quatro pares associados de formas (F) e caracteres (c) da Tabela 1 pode ser quantificada da seguinte maneira:

Tabela 1

Quantificação das contingências programadas de reforço.

\begin{tabular}{|c|c|c|c|c|c|}
\hline Formas & Caracteres & Formas & Caracteres & Formas & Caracteres \\
\hline $\mathbf{F} 1$ & $c 1 c 1 c 2 c 2 c 2$ & F1 & $\mathrm{c} 1 \mathrm{clc} 2 \mathrm{c} 2 \mathrm{c} 2$ & $\mathrm{Fl}$ & $c 1 c 1 c 2 c 2 c 2$ \\
\hline $\mathrm{I} 2$ & e2ceclelel & 12 & $c 2 \mathrm{c} 2 \mathrm{c} 1 \mathrm{c} 1 \mathrm{c}$ & 12 & $0202 c 1 c 1 \mathrm{c}$ \\
\hline 13 & $c 3 c 3 c 4 c 4 c 4$ & F3 & c3 $c 3 \times 4 \varepsilon 4 c 4$ & $\mathrm{F3}$ & $\mathrm{e} 3 \mathrm{c}: 3 \mathrm{c} 4 \mathrm{c} 4 \mathrm{c} 4$ \\
\hline F4 & $c^{4} c_{4} c^{3} 3 c^{3} c^{3}$ & F4 & $c 4 c 4 c 3 c, 3 c j$ & F4 & $c 4 c 4 c 3 c 3 c 3$ \\
\hline & $F M R$ & & Pos & & Forma \\
\hline
\end{tabular}


(a) Frequência Média de Reforço (FMR), obtida com base na divisão do número de respostas corretas possíveis em qualquer bloco de tentativas pelo número de respostas diferentes. A frequência média de reforço no exemplo é cinco, obtida pela divisão do número de respostas corretas possíveis nesta condição (20) pelo número de respostas diferentes (4); (b) probabilidade média programada de reforço para qualquer resposta dada uma forma (PRForma), obtida pela divisão da frequência média programada de reforço dada uma forma pelo número de respostas corretas possíveis dada uma forma. No exemplo PRForma é 0,5, obtida pela divisão entre a frequência média programada de reforço dada uma forma $(2,5)$ pelo número de respostas corretas possíveis dada uma forma (5); e (c) probabilidade média programada de reforço para qualquer resposta dada uma posição ( PRPos), obtida pela divisão da frequência média de reforço dada uma posição pelo número de respostas corretas possíveis dada uma posição (igual ao número de formas). A PRPos no exemplo é 0,25 , obtida pela divisão entre frequência média de reforço dada uma posição (1) e o número de respostas corretas possíveis (4). Oliveira-Castro et al. (1999) denominaram complexidade discriminativa as mudanças em FMR, PRForma e PRPos, as quais estão relacionadas às ocasiōes adequadas para emitir certos movimentos, em oposição a mudanças, por exemplo, no tipo e complexidade dos movimentos exigidos na tarefa, denominada complexidade topográfica.

A determinação das dimensões componentes da complexidade de tarefas e a especificação das condiçōes necessárias para a combinação entre as dimensões da tarefa na fixação de diferentes níveis de complexidade de tarefas poderia aplicar-se, em tese, a tarefas que variam em sua complexidade discriminativa e poderia contribuir para a especificação das condições ótimas de treino no ensino de tarefas com diferentes probabilidades programadas de reforço. Por exemplo, as relações entre sons da fala e letras do alfabeto carecem de sistematização quanto à quantidade de treino necessário para ensinar diferentes dificuldades da língua e aumentar o repertório verbal. Propostas instrucionais tentam estabelecer o treino mínimo de rela- ções que geram repertórios novos, não treinados e ensino de dificuldades da língua (Alessi, 1987; Goldstein \& Mousetis, 1989).

Hubner-D’Oliveira e Matos (1993) relataram três estudos com nove crianças entre quatro e cinco anos que investigaram a ocorrência de leitura sob controle textual de unidades menores (sílabas ou letras) utilizando o paradigma de equivalência. Após o treino do nome das figuras de BOCA, BOLA e BOTA (treino AB) e o nome de suas respectivas palavras escritas (treino $\mathrm{AC}$ ) foi testada a equivalência, onde a criança deveria emparelhar palavras e respectivas figuras (testes $\mathrm{BC}$ e $\mathrm{CB}$ ). As crianças obtiveram 100\% de acertos nos testes de equivalência $\mathrm{BC}$ e CB. A seguir, palavras novas (BALA, CABO e LATA) resultantes da recombinação das quatro sílabas de treino $(\mathrm{BO} / \mathrm{CA} /$ LA/TA) eram testadas para aferir controle por unidades menores (testes $\mathrm{B}^{\prime} \mathrm{C}^{\prime} \mathrm{e} \mathrm{C}^{\prime} \mathrm{B}^{\mathrm{c}}$ ). Contudo, apenas uma das quatro crianças mostrou $100 \%$ de acertos para todas as palavras. Hubner-D’Oliveira e Matos (1993) replicaram o estudo introduzindo montagem de anagramas das palavras e o ensino do nome da figura. O desempenho nos testes manteve-se ao acaso, embora o aumento do repertório (ensino de novo conjunto de palavras formado por COLA, LOBO e TOCO) tenha acarretado aumento do desempenho para dois dos três participantes. No terceiro estudo relatado por Hubner-D’Oliveira e Matos (1993) houve maior sistemática na variação da posição da sílaba nas palavras (BOCA, CABO, BOLO E LOBO). Resultados parciais mostraram "um controle por unidades menores melhor do que aquele observado quando o repertório aprendido era menor e a variação da posição silábica também era menor" (pp. 103-104). Investigação do efeito de oralização e cópia (dominós silábicos) sobre a leitura generalizada recombinativa realizado por Matos, Peres, HubnerD'Oliveira e Malheiros (1997) sugeriu que o treino simultâneo de oralização e cópia é mais eficiente do que o treino de cada uma destas dimensões isoladamente.

$\mathrm{Na}$ tentativa de estabelecer as condições necessárias à aquisição de controle por unidades menores, Rocha (1996) criou um tipo de linguagem artificial composta 
por quatro conjuntos de palavras dissílabas com caracteres arbitrários geradas por diferentes regras de formação. Tal como no estudo de Hubner-D’Oliveira e Matos (1993), foram treinadas as relações condicionais $\mathrm{AB}$ (palavras ditadas e respectivos desenhos) e $\mathrm{AC}$ (palavras ditadas e escritas) e testadas as relações $\mathrm{BC}$ e $\mathrm{CB}$ (palavras escritas e desenhos) e $\mathrm{B}^{\prime} \mathrm{C}^{\prime}$ e $\mathrm{C}^{\prime} \mathrm{B}$ ' (palavras escritas e desenhos de generalização). Condições de treino e respectivos testes foram denominadas conjuntamente de Fases. De acordo com as regras de formação, as palavras de treino da Fase 1 eram formadas com a mesma sílaba no início (bako, balo, bane, bafi); na Fase 2 eram formadas por sílabas diferentes (bena, niko, loke, lefi); na Fase 3 eram formadas por palavras terminavam com a mesma sílaba (befo, lifo, nafo, kefo); e na Fase 4 eram formadas por sílabas que eram usadas ora como primeira, ora como segunda sílaba em palavras diferentes (nibo, kani, bole e leka). A situação de teste consistiu de cinco tipos de recombinações: troca de letra na primeira sílaba, troca de letra na segunda sílaba, recombinação de sílaba, inversão de posição e todas as sílabas diferentes. Por exemplo, a recombinação das palavras de treino que iniciavam com a mesma sílaba resulta em bano, bilo, neba, kofi e kifa. Observou-se: (a) a emergência de relaçōes de equivalência (testes $\mathrm{BC}$ e $\mathrm{CB}$ ) para sete participantes nas quatro fases experimentais, e, em pelo menos três fases para cinco participantes e (b) emergência de leitura generalizada (testes $\mathrm{B}^{\prime} \mathrm{C}^{\prime} \mathrm{e} \mathrm{C}^{\prime} \mathrm{B}^{\circ}$ ) para sete dos 12 participantes. Contudo, segundo Rocha (1996), não foi possível determinar quais elementos dos estímulos compostos passaram a controlar respostas generalizadas (sílabas, fonemas, vogais, consoantes).

Segundo Oliveira-Castro (2000), a análise da complexidade discriminativa das tarefas possibilitaria a quantificação da diversidade de treinos, identificação dos efeitos de tal complexidade sobre a emergência de leitura generalizada e comparação das características da situação de treino com aquelas da situação de teste. Por exemplo, dado que no procedimento utilizado por Rocha (1996), para cada letra (forma arbitrária) somente um fonema era reforçado, FMR, a probabilidade programada de reforço para as palavras (PRPal) e PRPos foram calculadas, seguindo a mesma forma de cálculo de FMR, PRPos e PRForma, e observou-se diferenças na complexidade discriminativa das palavras de treino das Fases: as Fases 1 e 3 foram idênticas (FMR=1,78; PRPal = 0,25; PRPos = 0,65) e menores do que a complexidade discriminativa das Fases 2 $(\mathrm{FMR}=1,78 ; \mathrm{PRPal}=0,25 ; \mathrm{PRPos}=0,35)$ e $4(\mathrm{FMR}=$ 2,00; PRPal =0,25; PRPos =0,25). Segundo OliveiraCastro (2000), os resultados dos testes de nomeação das palavras de treino nas quatro Fases de Treino 1 a 4 (88\%, $87 \%, 75 \%, 75 \%$ de acertos) parecem refletir as diferenças de complexidade. Oliveira-Castro (2000) calculou a semelhança funcional entre as situações de treino e teste comparando a porcentagem de acertos na leitura de cada fonema em determinada posição (por exemplo, a letra "O" na segunda ou terceira posiçôes) e com determinada letra adjacente (por exemplo, "BO") na situação de teste com a probabilidade programada de reforço para o mesmo fonema e com a mesma letra adjacente (PRLetra), no treino. Análise de regressão múltipla indicou efeito, significativo estatisticamente, de PRPos e PRLetra tal que aumentos nos valores destas variáveis acarretaram aumentos $\mathrm{da}$ porcentagem de acertos nos testes, explicando 31\% da variância da porcentagem de acertos. Portanto, quanto mais semelhantes forem as condições de treino e teste, melhor será o desempenho; a quantificação das contingências programadas de reforço possibilitaria a mensuração do grau de semelhança entre estas condições.

O presente trabalho teve como objetivo a quantificação das contingências programadas de reforço tanto nas sessões de treino quanto nas sessões de recombinação de estímulos e respostas que formavam pares associados. A quantificação consistiu em considerar "não-correspondência" e "correspondência" entre características dos estímulos e respostas que compuseram os pares associados em termos de probabilidade programada de reforço, a qual pode variar entre 0,00 e 1,00. Este tipo de quantificação da relação forma/caractere é análogo à relação grafema/fonema e viria contribuir para a substituição de classificaçôes qualitativas relacionadas à 
pronúncia ou acentuação das palavras e ofereceria melhor especificação da quantidade de treino (área da função ou tempo total estimado para diminuição das respostas precorrentes auxiliares) necessário para ensinar diferentes dificuldades da língua.

\section{MÉTODO}

\section{Participantes}

Participaram do experimento doze alunos da Universidade de Brasília, três homens $(M=18,00$ anos; $D P=$ $1,00)$ e nove mulheres ( $M=18,44$ anos; $D P=1,42$ ), os quais receberam pontos em disciplina proporcionalmente às horas de participação na pesquisa. Sete alunos eram do curso de psicologia e os demais eram de outros cursos.

\section{Material}

O experimento foi integralmente realizado em um computador e o programa, desenvolvido para o ambiente Windows 95, permitiu tanto a composição e seleção dos caracteres utilizados no experimento quanto o registro de tempo, frequências, acertos e erros.

\section{Procedimento}

Antes de iniciar a sessão experimental, foram apresentadas aos participantes as seguintes instruçōes na tela do computador :

Agradecemos a sua participação neste experimento sobre memória. A sua tarefa consiste em memorizar alguns códigos, cada um associado a um símbolo. Leia com atenção as instruções a seguir. Um símbolo aparecerá numa tela azul. Clique com o mouse em "AUXILIO" para ver o código correspondente ao símbolo; quando estiver pronto para escrever os códigos, clique em “SAIR”. Para escrever o código, digite um componente do código de cada vez. Atenção ! você não poderá corrigir as suas respostas. Se você digitar um componente errado, continue. Digite o próximo componente que compõe o código. Se todos os componentes do código estiverem corretos, aparecerá a palavra
“CORRETO !” na parte inferior da tela. Se qualquer um dos componentes estiver errado, aparecerá a palavra “ERRADO!" Os símbolos se repetirão no decorrer da sessão e estarão sempre associados aos mesmos códigos. A sua tarefa, portanto, é memorizar o código correspondente a cada símbolo. Você poderá usar o “AUXILIO” sempre que julgar necessário. Porém, cada vez que você escrever, com o mouse, um código corretamente sem o "AUXILIO" você ganhará um ponto no marcador à esquerda da tela. Após a apresentação da palavra “CORRETO !” ou “ERRADO!”, um novo símbolo será apresentado, e você deverá seguir os mesmos passos descritos acima. Clique em OK para começar.

Um bloco de tentativas foi definido como a apresentação de nove pares de formas e caracteres. Antes de realizar a primeira sessão, dois pares de formas arbitrárias eram apresentados duas vezes como exemplos. Após estes exemplos o participante deveria pressionar com o mouse a tecla "OK" para iniciar a sessão. A sequência de eventos durante a aprendizagem de cada par era: 1) apresentação de uma forma arbitrária na parte superior da tela azul, sob a palavra símbolo; 2) se o participante pressionasse a palavra "auxílio", utilizando o mouse, a palavra "aguarde" aparecia na tela azul por 0,5 segundo $(\mathrm{t} 1) ; 3)$ após $\mathrm{t} 1$, um código composto de três formas era apresentado na parte central de uma tela branca; 4) se o participante pressionasse a tecla "SAIR", utilizando o mouse, a palavra "aguarde" aparecia na tela azul por 0,5 segundo (t2); 5) a tela azul reaparecia com o cursor no início do retângulo sob a palavra código; 6) o participante digitava, usando o mouse, a sequência de três caracteres constituintes do código; 7) após 0,5 segundo, a palavra “CORRETO!” ou “ERRADO!” era apresentada, dependendo dos caracteres digitados; 8) ocorria um intervalo de 0,5 segundo; e 9) uma nova forma arbitrária aparecia iniciando outra aprendizagem entre formas e caracteres arbitrários. Os passos de 2) a 6) poderiam ser abreviados se o participante digitasse a seqüência de caracteres corretamente sem consultar o 
"auxílio". A sessão terminava segundo os seguintes critérios: após a apresentação de 24 tentativas ou após o participante digitar corretamente todos os nove caracteres correspondentes às nove formas em duas tentativas consecutivas sem consultar o auxílio ou, por fim, após realizar a tarefa durante, aproximadamente, quarenta minutos.

\section{Delineamento}

O experimento utilizou um delineamento experimental de medidas repetidas, isto é, os participantes foram submetidos a todas as condiçõos experimentais. A ordem de apresentação das condições experimentais aos participantes foi variada afim de possibilitar que as condiçôes fossem apresentadas em diferentes seqüências, excetuando as Condições 1 e 4 , as quais corresponderam sempre às Sessões 1 e 4 , respectivamente. O conjunto de formas e caracteres utilizados nas seis condiçôes foi relacionado às diversas condições, excetuando os Conjuntos 1 e 4, os quais estiveram sempre relacionados às Condiçôes 1 e 4 , respectivamente.

As condiçōes experimentais foram compostas por nove pares associados de formas-códigos. Duas dimensões da tarefa de par associado foram alteradas no presente experimento: as posições possíveis de um caractere numa sequência de respostas e a quantidade de caracteres. Utilizando o procedimento de pares associados de formas arbitrárias (e.g., o estímulo composto "F1F2" e as três respostas "c1c8c8"), numa condição experimental onde posições ocupadas pelo caractere foi manipulada, a probabilidade programada de reforço para uma resposta foi 1,00 , isto é, uma das formas (e.g., F1) correspondeu a um determinado caractere (e.g., c1, c2 ou c3) e a outra forma (e.g., F2) correspondeu a uma das três posições possíveis ocupadas pelos caracteres (e.g., "c1c0c0, c0c1c0, ou c0c0c1"). Em outra condição, a probabilidade programada de reforço para uma resposta, dada uma forma específica, foi 0,33, isto é, uma das formas (e.g., F1) correspondeu aos três caracteres possíveis (e.g., c1, c2 e c3) e a outra forma (e.g., F2) correspondeu às três posições possíveis ocupadas pelos diferentes caracteres (e.g., "c1c0c0, c0c1c0 e c0c0c1").
Numa condição experimental onde quantidade de caracteres foi manipulada, a probabilidade programada de reforço para uma resposta, dada uma forma específica, foi 1,00, isto é, uma das formas (e.g., F7) correspondeu a um determinado caractere (e.g., c7, c8 ou c9) e a outra forma (e.g., F10) correspondeu a um determinado número de repetiçôes do caractere (e.g., "c9c0c0, c9c9c0 ou c9c9c9"). Em outra condição, a probabilidade programada de reforço para uma resposta, dada uma forma específica, foi 0,33, isto é, uma das formas (e.g., F7) correspondeu aos três caracteres possíveis (e.g., c7, c8 e c9) e a outra forma (e.g., F10) correspondeu às três repetições possíveis dos diferentes caracteres (e.g., "c9c0c0, c9c9c0 e c9c9c9").

Após o treino dos dois tipos de correspondência forma/caractere tanto para posição quanto para quantidade, foi testada a aprendizagem das relações de posição e quantidade sob os dois tipos de probabilidades programadas de reforço, 0,33 e 1,00 (doravante, Condição REC 0,33 e Condição REC 1,00) em sessões de recombinação, que diferiam das sessōes de treino em dois aspectos: a) eram utilizados, na composição dos estímulos e respostas dos pares associados das sessōes de recombinação, caracteres diferentes daqueles das condiçōes Prob 1,00 e Prob 0,33; além disso, b) tais caracteres eram aprendidos em uma sessão de treino com valor de probabilidade programada de reforço 0,67 , valor intermediário entre probabilidade programada de reforço 0,33 e 1,00. A Condição REC 1,00 permitiu determinar o tempo total estimado de diminuição da resposta precorrente auxiliar, i.e., a área da Equação 1 para aprender pares associados cuja característica do estímulo (uma forma arbitrária, por exemplo) predissesse uma determinada característica da resposta (a primeira posição a ser ocupada por um caractere, por exemplo), a qual era formada por caracteres diferentes daqueles do treino na Condição Prob 1,00. A Condição REC 0,33 permitiu determinar a área para aprender pares associados cuja característica do estímulo predissesse várias características da resposta (as três posições possíveis de serem ocupadas por caracteres, por exemplo), a qual era formada por 
caracteres diferentes daqueles do treino na Condição Prob 0,33 . A predição era que diminuição no valor de probabilidade de 1,00 para 0,33 acarretaria aumento no tempo total para aprendizagem.

Em termos de complexidade, as Condiçōes Prob 1,00, Prob 0,33, REC 1,00 e REC 0,33 tiveram os mesmos valores de frequência média de reforço $(27 / 4=6,75)$; probabilidade média programada de reforço para qualquer resposta, dada uma forma $(\{3 / 2\} / 3=0,50)$ e probabilidade média programada de reforço para qualquer resposta, dada uma posição $(\{9 / 4\} / 9=0,25)$. Prob0,67 teve valor de frequência média de reforço $(27 / 6=4,5)$; probabilidade média programada de reforço para qualquer resposta, dada uma forma $(\{3 / 2\} / 3=0,50)$ e probabilidade média programada de reforço para qualquer resposta, dada uma posição $(\{9 / 3\} / 9=0,33)$.

A duração total estimada da resposta precorrente auxiliar/corretas (área da Equação 1) foi a variável dependente principal. As outras variáveis dependentes medidas no presente trabalho foram a duração total de latência da resposta/corretas nos blocos de tentativas, isto é, a soma total dos intervalos de tempo entre a apresentação do estímulo (forma arbitrária) e o início da resposta de digitar os caracteres, dividida pelo número de respostas nos blocos de tentativas; e a duração total do tempo de resposta/ correta nos blocos de tentativas, isto é, a soma total dos intervalos de tempo entre o início e a conclusão da digitação dos caracteres com o mouse, dividida pelo número de respostas nos blocos de tentativas.

\section{Resultados E Discussão}

A partir da Equação 1, calculou-se a área $\left(b^{2} / 2 a\right)$ para cada participante, em cada condição experimental. A Figura 1 mostra os valores de área para cada participante em cada condição experimental. Observa-se o decréscimo no valor de área das duas condições de treino (Prob 1,00 e Prob 0,33) para as respectivas condições de Recombinação (REC 1,00 e REC 0,33): para 10 participantes na Condição Prob 1,00 e para nove participantes na Condição Prob 0,33. O valor de área na Condição Prob 1,00 foi menor $(M=0,98 ; \mathrm{DP}=0,42)$ do que o na Condição Prob $0,33(\mathrm{M}=1,63 ; \mathrm{DP}=0,66)$ para 10 participantes. Em relação às condiçôes de recombinação, o valor de área na Condição REC 1,00 foi menor ( $\mathrm{M}=0,36 ; \mathrm{DP}=0,25)$ do que o na Condição REC 0,33 (M=1,44; DP= 0,83) para todos os participantes.

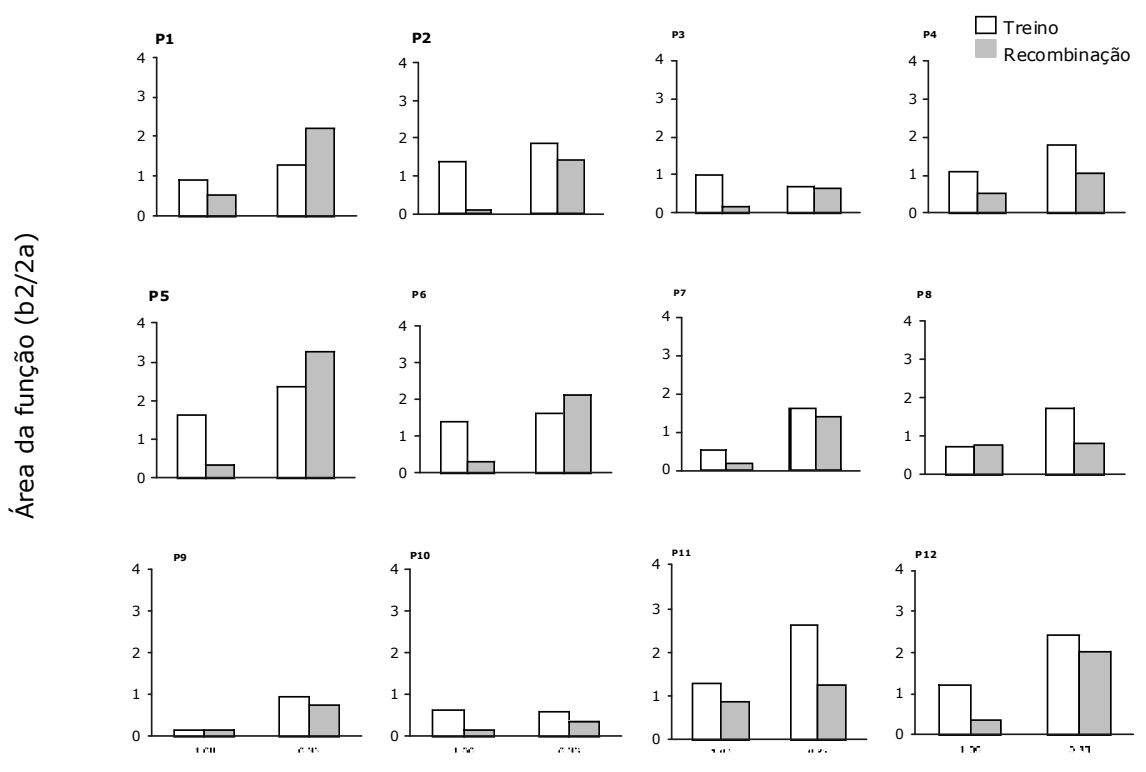

Figura. 1. Área da função para os 12 participantes nas condiçóes Prob 1,00 e Prob 0,33 no treino e recombinação. 
ANOVA para medidas repetidas foi conduzida com o objetivo de comparar os valores de área como uma função dos fatores intra-sujeito Condição (1 a 4) e Participante, e dos fatores entre-sujeito Conjunto (1 e 2) e Sessão (1 e 2). A ANOVA indicou efeito significativo estatisticamente $($ alfa $=0,05)$ de Condição, $F(3,7)=18,95, \mathrm{p}=$ 0,0001, e Participante, $\mathrm{F}(11,7)=3,97, \mathrm{p}=0,001$, e efeitos não-significativos de Sessão, $\mathrm{F}(1,7)=1,340 \mathrm{p}=$ 0,256 , e Conjunto, $\mathrm{F}(1,7)=1,186 \mathrm{p}=0,285$. Teste Tukey para múltiplas comparações foi conduzido e mostrou diferenças significativas (alfa $=0,05$ ) entre as Condições Prob 1,00 e REC 1,00 ( $\mathrm{p}=$ 0,026), Prob 1,00 e Prob 0,33 (p = 0,027) e REC 1,00 e REC 0,33 ( $\mathrm{p}=$ $0,0001)$ e diferenças não-significativas entre Prob $0,33 \mathrm{e}$ REC 0,33 ( $\mathrm{p}=0,378)$. Estes resultados sugerem que, apesar das diferenças individuais significativas entre os participantes, diminuição no valor de probabilidade programada de reforço de 1,00 para 0,33 acarretou aumento no tempo total estimado de resposta precorrente auxiliar tanto no treino quanto nas sessões de recombinação. A diferença observada entre as Condições Prob 1,00 e Prob 0,33 pode ser atribuída aos diferentes valores de probabilidade programada de reforço utilizados na quantificação das características de estímulos e respostas da tarefa de par associado utilizada no treino e em sessão de recombinação.

ANOVA para medidas repetidas foi conduzida com o objetivo de comparar os valores de soma total de duração de latência/corretas como uma função de Condição, Participante (fatores intra-sujeitos), Conjunto e Sessão (fatores entre-sujeitos). A ANOVA indicou efeito significativo estatisticamente (alfa $=0,05)$ de Condição, $\mathrm{F}(3,7)=21,80, \mathrm{p}=0,0001$, Participante $\mathrm{F}(11,7)=$ $2,75 \mathrm{p}=0,013$, Sessão, $\mathrm{F}(1,7)=0,049 \mathrm{p}=4,20$, e Conjunto, $F(1,7)=0,855 p=0,034$. Teste Tukey para múltiplas comparações foi conduzido e mostrou diferenças significativas (alfa $=0,05)$ entre Prob 0,33 e REC 0,33 ( $\mathrm{p}=0,019)$, Prob 1,00 e Prob 0,33 ( $\mathrm{p}=0,0001$ ) e entre REC 0,33 e REC 1,00 ( $\mathrm{p}=0,0001)$ e diferenças não-significativas entre as Condiçôes Prob 1,00 e REC $1,00(\mathrm{p}=0,141)$. Estes resultados sugerem que, apesar das diferenças individuais significativas entre os participantes, a diminuição no valor de probabilidade programada de reforço de 1,00 para 0,33 , produziu aumentos na duração de latência de resposta/corretas.

ANOVA para medidas repetidas foi conduzida com o objetivo de comparar os valores de soma total de duração de tempo de resposta/corretas como uma função das Condição, Participante (fator intra-sujeitos), Conjunto e Sessão (fatores entre-sujeitos). A ANOVA indicou efeito significativo estatisticamente $(a l f a=0,05)$ de Condição, $\mathrm{F}(3$, 7) $=14,13, p=0,0001$, e efeitos não-significativos de Conjunto $F(1,7)=1,92 \mathrm{p}=0,176$, Participante $\mathrm{F}(1,7)$ $=1,91 \mathrm{p}=0,077$, e Sessão $\mathrm{F}(1,7)=2,26 \mathrm{p}=0,143$. Teste Tukey para múltiplas comparaçôes foi conduzido e mostrou diferenças significativas (alfa $=0,05)$ entre as Condições Prob 1,00 e Prob 0,33 ( $\mathrm{p}=$ 0,005), e REC 1,00 e REC0,33 ( $p=0,001$ ) e diferenças não-significativas entre Prob 1,00 e REC 1,00 ( $\mathrm{p}=0,075)$ e Prob 0,33 e REC $0,33(\mathrm{p}=0,233)$. Em suma, diminuição no valor de probabilidade programada de reforço de 1,00 para 0,33 acarretou aumento no valor de tempo de resposta/corretas entre as duas condições de treino e recombinação, porém não acarretou aumento entre cada condição de treino e sua respectiva condição de recombinação.

Os resultados sugerem que a análise em termos de contingências programadas de reforço possibilitou a quantificação do tipo de correspondência entre a forma (que indicou posição ou quantidade) e a variação do caractere (em termos de posição ou quantidade de repetições) nas Condições Prob 0,33 e Prob 1,00, respectivamente. A análise em termos de contingências programadas de reforço forneceu um modo de quantificar os diferentes tipos de treinos e permitiu comparar o grau de semelhança entre as sessões de treino e de recombinação. Nas sessões de treino, aumento no valor de probabilidade programada de reforço de 0,33 para 1,00 acarretou a diminuição no valor de área da função, latência/corretas e tempo de resposta/corretas. Nas sessôes de recombinação, observou-se padrão semelhante ao do treino, aumento no valor de probabilidade programada de reforço de REC 
0,33 para REC 1,00 acarretou a diminuição no valor de área da função, latência/corretas e tempo de resposta/corretas para todos os participantes.

A extensão da análise em termos de contingências programadas de reforço, utilizando o procedimento de pares associados como medida precorrente auxiliar, pode incluir situações análogas ao treino de cópia ou transcrição de palavras formadas com grafemas conhecidos, onde ocorre variação de posição e/ou adjacência dos caracteres treinados. A quantificação das contingências programadas de reforço poderia auxiliar tanto na especificação da variação sistemática de sílabas e letras necessárias para a emergência de leitura generalizada quanto na quantidade de treino necessária para ensinar este tipo de dificuldade da língua.

\section{REFERÊNCIAS}

Alessi, G. (1987). Generative strategies and teaching for generalization. The Analysis of Verbal Behavior, 5, 1527.

Fleishman, E. (1975). Toward a taxonomy of human performance. American Psychologist, 30, 1127-1149.
Goldstein, H., \& Mousetis, 1. (1989). generalized language learning by children with severe mental retardation: Effects of peers expressive modeling. Journal of Applied Behavior Analysis, 22, 245-259.

Matos, M.A., Peres, W., Hubner-D'Oliveira, M.M. \& Malheiros, R.H.S. (1997). Oralização e cópia: efeitos sobre a aquisição de leitura generalizada recombinativa. Temas em Psicologia, 1, 47- 64.

Hubner-D'Oliveira, M.M. \& Matos, M.A. (1993). Controle discriminativo na aquisição da leitura: efeito da repetição e variação na posição das sílabas e letras. Temas em Psicologia, 2, 99-108.

Oliveira-Castro, J.M. (2000). Contingências programadas de reforço e complexidade discriminativa de tarefa: aplicações a situaçôes de ensino. Em R. C. Welenska (Org.) Sobre comportamento e cognição, vol. 6, Santo André: Set.

Oliveira-Castro, J.M., Coelho, D.S., \& e OliveiraCastro, G.A. (1999). Decrease of precurrent behavior as training increase: Effects of task complexity. Psychological Record, 49, 299-325.

Rocha, A. M. (1996). Variação da composição dos estimulos treinados e desenvolvimento de controle por unidades textuais minimas. Dissertação de mestrado não publicada. Universidade de Brasília.

Wood, R.E. (1986). Task complexity: Definition of the construct. Organizational Behavior and Human Decision Process, 37, 60-82. 